\section{Discussion}

Several biologic sealants are now available for use in cardiovascular surgery, with the best studied being gelatin-resorcin-formalin (GRF) glue and a 2-component fibrin sealant. The use of GRF glue has been associated with tissue necrosis, with destruction of the aortic media causing vascular wall thinning and rupture. ${ }^{2}$ Its use has also been linked with coronary ostial stenosis and fatal cerebral embolism. ${ }^{3}$ Bioglue, which is composed of bovine serum albumin (45\%) and gluteraldehyde (10\%), is marketed as being less tissue toxic than GRF (gluteraldehyde 37\%), and initial clinical experience was reported by Raanani and colleagues ${ }^{4}$ in 2001 . Polymerization occurs when the 2 components are mixed, reaching bonding strength within 2 minutes. This tissue glue is also described as being more liquid in consistency than the GRF glue. Its potential to penetrate the intravascular circuit is thus higher. Thus it has the potential to embolize, as in the case described. The emboli then activates the clotting cascade directly and also produces stasis, which is one of the triad described by Virchow necessary for thrombus formation and propagation.
This case also illustrates the importance of the use of postmortem studies to elucidate the cause of death, as well as the use of digital imaging by the pathologists, to demonstrate the postmortem findings, obviating the need for tissue retention. ${ }^{5}$

\section{References}

1. Koehnlein HE, Lemperle G. Experimental studies with a new gelatinresorcin-formaldehyde glue. Surgery. 1969;66(2):377-82

2. Fukunaga S, Karck M, Harringer W, Cremer J, Rhein C, Haverich A. The use of gelatin-resorcin-formalin glue in acute aortic dissection type A. Eur J Cardiothorac Surg. 1999;15:564-70.

3. Mastroroberto P, Chello M. Embolization of biological glue after repair of acute aortic dissection. Ann Thorac Surg. 1996;62:946-7.

4. Raanani E, Latter DA, Errett LE, Bonneau DB, Leclerc Y, Salasidis GC. Use of 'bioglue' in aortic surgical repair. Ann Thorac Surg. 2001;72: $638-40$.

5. Guidelines on autopsy practice. Report of the working group of the Royal College of Pathologists, September 2002. www.rcpath.org.

\title{
A technique for repair of mitral paravalvular leak
}

\author{
Abeel A. Mangi, MD, and David F. Torchiana, MD, Boston, Mass
}

$\mathrm{T}$ he incidence of paravalvular leak 15 years after mitral valve replacement (MVR) approximates 17\%. ${ }^{1,2}$ Its relative incidence after mechanical and bioprosthetic replacement is debated. ${ }^{1}$ Running monofilament suture techniques $^{3}$ and MVR for endocarditis ${ }^{4}$ have been implicated in the development of paravalvular leaks. Twenty-two percent of patients with paravalvular leaks are diagnosed in the first week after MVR, and another 52\% are diagnosed within the first postoperative year. Patients requiring operative repair are older, have symptomatic heart failure, hemolytic anemia, and larger leaks. Surgical intervention to repair the leak improves symptoms of congestive heart failure, augments the hematocrit value, decreases the need for blood transfusion, and is an independent predictor of long-term survival when compared with medical therapy. ${ }^{4}$ Choice of operation involves either direct suture repair of the leak site, which carries a failure rate of $13 \%$; or replacement of the valve, which carries a failure rate of up to $35 \%$ and poses a technical

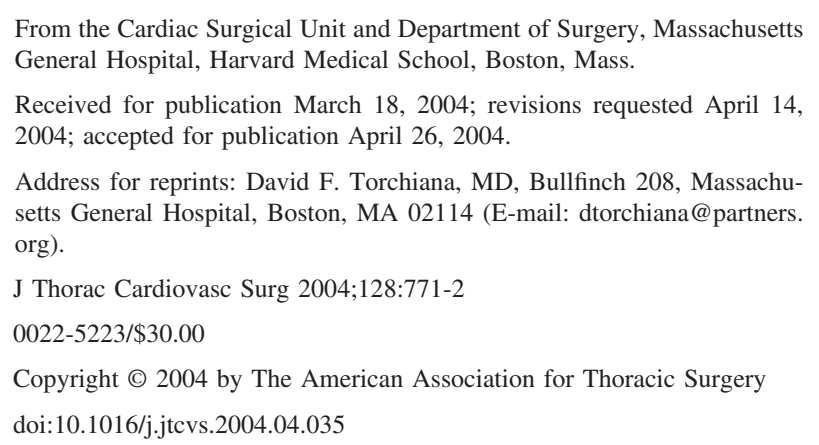

challenge. Here we propose a simple technique for repair of selected mitral paravalvular leaks that incorporates healthy, fullthickness autologous tissue into the repair and has promising durability.

\section{Technique}

The operation is conducted during continuous intraoperative transesophageal echocardiography (TEE) and commences with standard ascending aortic and bicaval cannulation. After the heart is arrested with antegrade intermittent cold blood cardioplegia, a standard superior septal incision is made. If both atria are significantly enlarged, the prosthesis can be approached solely through the interatrial septum. This approach yields excellent exposure of the anterior part of the mitral prosthesis that abuts the interatrial septum and is proximate to the posteromedial commissure of the native mitral valve. In our experience this location accounts for $38 \%$ of mitral paravalvular leaks. ${ }^{5}$ Although TEE accurately identifies $88 \%$ of mitral paravalvular leaks, ${ }^{5}$ clear communication between the surgeon and echocardiographer is essential to ensure that the location and number of leaks are accurately defined, especially because nomenclature in this area can be confusing. As an example, the location that we are discussing is on the anterior aspect of the mitral prosthesis but also is proximate to the posteromedial commissure of the native mitral valve. It is therefore critical that the echocardiographer and the surgeon refer to standardized reference points. Depending on the extent of the leak, the first pledget-supported, braided polyester stitch is placed as a horizontal mattress stitch through a fold of the left atrial wall and is then brought directly into the sewing ring at the most posterior aspect of the leak near the coronary sinus. The remaining sutures 


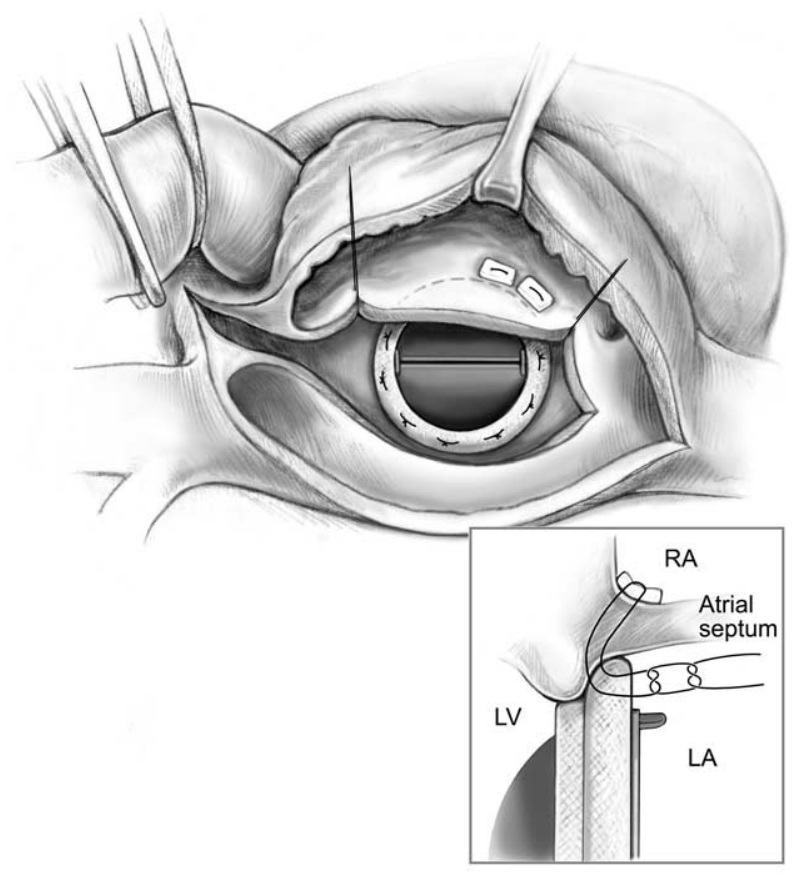

Figure 1. Demonstration of suture technique incorporating healthy atrial septal tissue adjacent to the sewing ring for repair of mitral paravalvular leak. $R A$, Right atrium; $L V$, left ventricle; $L A$, left atrium.

are placed in similar fashion through the interatrial septum from the right side to the left and then directly though the sewing ring (Figure 1). Up to 5 sutures might be needed to completely obliterate the leak. The left atrium is closed with a continuous 4-0 Prolene suture (Ethicon, Inc, Somerville, NJ) starting on the atrial dome and suturing caudad onto the interatrial septum. The right atrium is closed after removal of the aortic clamp.

\section{Discussion}

We have performed this operation on 2 patients with anterior paraprosthetic leaks after MVR. The first patient is a 50-year-old man who had undergone aortic valve replacement and MVR at age 14 years. At age 32 years, his valves were replaced with Starr-
Edwards prostheses (Baxter Healthcare Corp, Edwards Division, Santa Ana, Calif) for endocarditis. He had congestive heart failure 15 years later and was found to have a large paravalvular leak and a severely calcified mitral annulus. Attempted treatment with a percutaneously delivered clamshell device marginally improved the degree of regurgitation but caused worse hemolysis. The second patient is a 78-year-old woman who had undergone placement of a St Jude Medical prosthesis (St Jude Medical, Inc, St Paul, Minn) 12 years before for rheumatic mitral stenosis and regurgitation. She presented in New York Heart Association class III heart failure with a hemolytic anemia. At the time of operation in both cases, the majority of the prosthesis was endothelialized, and the sewing ring appeared to be largely incorporated into the atrial endocardium. However, approximately $25 \%$ of the sewing ring was bare. This area corresponded to the paravalvular leak seen on TEE, as described above. Repair of the leak was undertaken in both cases with the apposition of healthy, full-thickness, atrial septal tissue against the denuded sewing ring of the valve by using the technique described. Both patients remain free of paravalvular leak and mitral regurgitation 4 and 5 years after repair, respectively. This simple strategy appears to offer a durable solution to the clinical problem of paravalvular leak in select instances in which the leak is located anteriorly near the interatrial septum.

\section{References}

1. Hammermeister K, Sethi GK, Henderson WG, Grover FL, Oprian C, Rahimtoola SH. Outcomes 15 years after valve replacement with a mechanical versus a bioprosthetic valve: final report of the Veteran's Affairs Randomized Trial. J Am Coll Cardiol. 2000;36(4):1152-8.

2. Jindani A, Neville EM, Venn G, Williams BT. Paraprosthetic leak: a complication of cardiac valve replacement. J Cardiovasc Surg. 1992; 32:503-8.

3. Dhasmana JP, Blackstone EH, Kirklin JW, Kouchoukos NT. Factors associated with periprosthetic leakage following primary mitral valve replacement: with special consideration of the suture technique. Ann Thorac Surg. 1983;35:170-8.

4. Genoni M, Franzen D, Vogt P, Seifert B, Jenni R, Kunzli A, et al. Paravalvular leak after mitral valve replacement: improved long-term survival with aggressive surgery? Eur J Cardiothorac Surg. 2000;17: 14-9.

5. Foster GP, Isselbacher EM, Rose GA, Torchiana DF, Akins CW, Picard MH. Accurate localization of mitral regurgitant defects using multiplane transesophageal echocardiography. Ann Thorac Surg. 1998;65(4): 1025-31. 